\title{
A Transient Analysis of Heat Transfer in Double Stack Cold Plates
}

\author{
Ron J. Pieper and Allan D. Kraus \\ Department of Electrical and Computer Engineering \\ Naval Postgraduate School, Monterey, California 93943
}

\begin{abstract}
There is practical interest in the mathematical characterization of the transient performance of heat dissipating extended surfaces to problems in electronic packaging. Although there has been a significant amount of work concentrating on predicting transient behavior in basic monolithic fin structures and shapes, none of these investigations is capable of handling complicated fin structures such as single or double cold-stack plates. As an example illustrating the application of the two-port method for transient analysis, the transient response to a step increase in power dissipation is predicted.
\end{abstract}

\section{Introduction}

It is well known that the reliability and performance of electronic components is highly dependent on operating temperatures. In situations where the components can be selectively turned on/off or in situations where the cooling system behaves erratically or has a catastrophic failure, the thermal transients involved could lead to conditions untenable to the normal operation of one or more electronic components. Cold plate design can play an important part in cooling electronic packages within the temperature limits specified.

Because time varying boundary conditions in extended surfaces must be handled on a case by case basis, the thrust here is to provide a methodology for characterizing the fin, which is independent of the specific time variation in the boundary conditions. A matrix characterization of fins which is independent of the form of the boundary condition has already been reported in the literature for the static (time independent) case [1].

\section{Background}

Because the heat dissipating structures of interest are composed of fins which are assumed to satisfy the Murray-Gardner conditions [1], a one-dimensional heat transfer analysis is adequate. The heat flow can be defined:

$$
q=k A \frac{d T}{d x}
$$

where the coordinate $x$ is taken opposite to the direction of positive heat flow, $A$ is the cross-sectional area, $q$ is the heat flow (analogous to current), and $T$ is the temperature (analogous to voltage). Figure 1 represents an extended surface rectangular fin of height $b$, length $L$, and thickness $\delta$. The tip $(x=0)$ and base $(x=b)$ are the designated boundaries. In the analysis to follow, some standard assumptions are made.

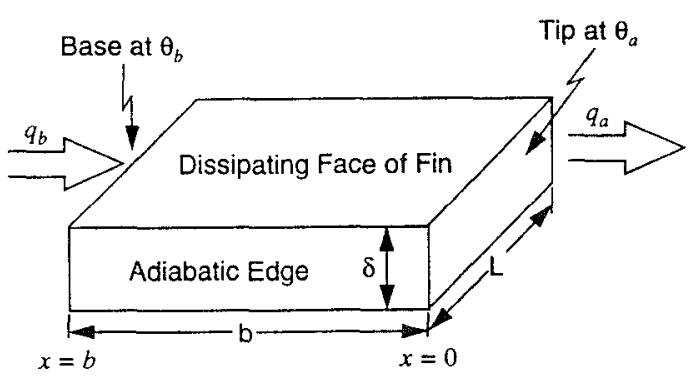

Figure 1: Rectangular fin showing significant physical and dimensional parameters.

The material used in the construction of the fin is characterized by a uniform conductivity, $k$, and uniform specific heat, $c$ and density, $\rho$. It is also assumed that the heat transfer coefficient due to convection, $h$, is also known. The environment temperature $T_{e}$ will be controlled by a forced-air cooling system. Examination of the energy balance condition

$$
\frac{d^{2}}{d x^{2}} \theta(x, t)-m^{2} \theta(x, t)=\frac{1}{\alpha} \frac{d \theta}{d t}
$$




\section{Transient Analyses of the Dou- ble Stack Cold Plate}

As illustrated in Fig. 2, the entire cold plate structure is assumed to be composed of a large number of repeating sections. This basic structure, which is identified within the box shown on the figure, is taken to be far enough from the vertical extremities of the double stack cold plate to justify ignoring end effects. Assuming a large number of such repeating sections $\left(N_{f} \geq 20\right)$, the overall performance of the double stack cold plate can be approximately predicted by appropriately scaling the performance of the basic repeating section being analyzed. The assumption of the approximate equivalence of the sections then forces the boundary condition that there is no steady state heat flow, i.e., $q=0$, across the conducting surfaces between repeating sections. This specifically includes the exterior cover plate surfaces and, for later reference, the splitter plate surfaces.

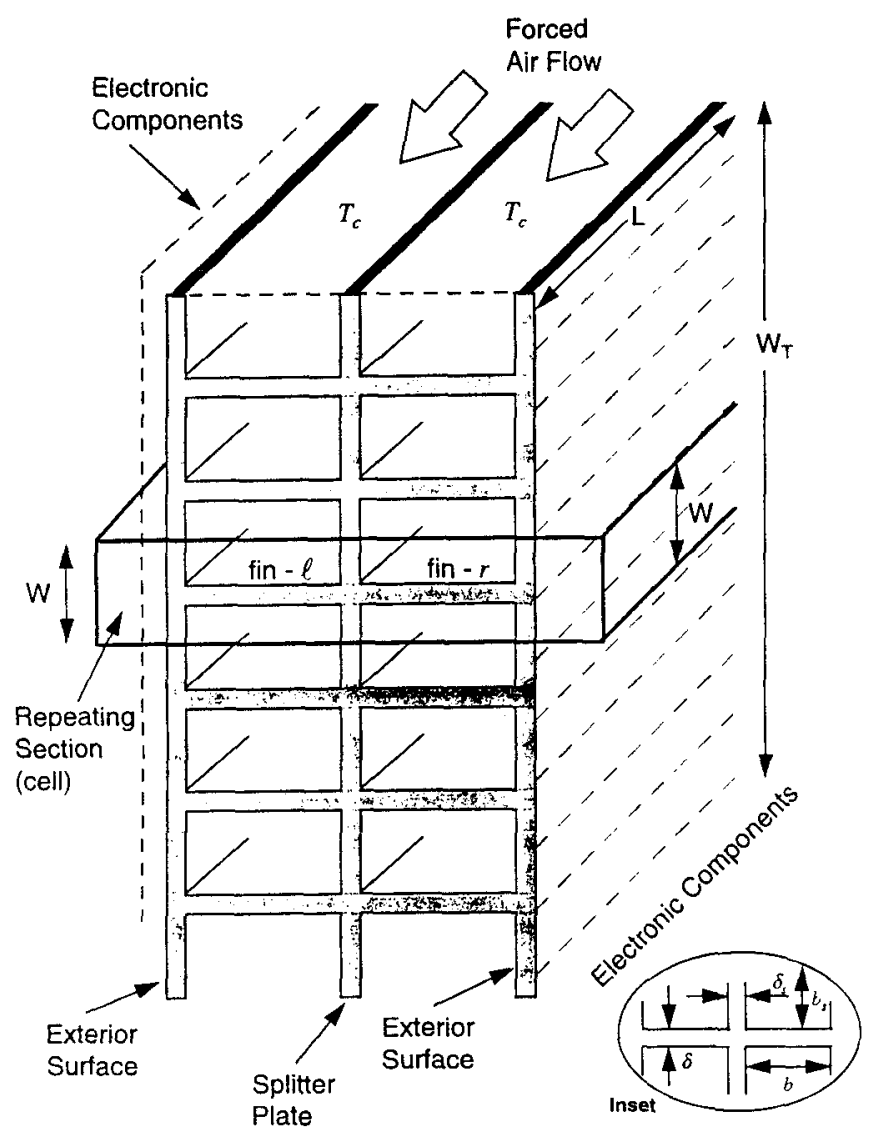

Figure 2: Artist's conception for electronic package with double stack cooling design.

Finally, it is observed that the left and right connecting structures between exterior surfaces and split- ter plates are identifiable as fin- $r$ and fin- $\ell$, respectively. The comprehensive static analysis of this structure covering all regimes has been completed [3] (see Table 1). Due to length limitations the final results for the static analysis will be summarized here. Excess cover plate temperatures can be related to the power dissipation requirements via

Table 1: Static Analysis Model [3]

$$
\begin{aligned}
& z_{11}=z_{22}=\frac{\left((\gamma+2) \Lambda^{4}-\gamma+2\right) z_{0}}{(\gamma+2) \Lambda^{4}+2 \gamma \Lambda^{2}+\gamma-2} \\
& z_{12}=z_{21}=\frac{4 \Lambda^{2} Z_{0}}{(\gamma+2) \Lambda^{4}+2 \gamma \Lambda^{2}+\gamma-2} \\
& f_{11}=f_{22}=\frac{\left(h S z_{11}+1\right) z_{11}-h S z_{12}^{2}}{\left(h^{2} S^{2} z_{11}+h S\right) z_{11}-h^{2} S^{2} z_{12}^{2}+h S z_{11}+1} \\
& f_{12}=f_{21}=\frac{z_{12}}{\left(h^{2} S^{2} z_{11}+h S\right) z_{11}-h^{2} S^{2} z_{12}^{2}+h S z_{11}+1}
\end{aligned}
$$

$$
\left[\begin{array}{l}
\theta_{b \ell} \\
\theta_{b r}
\end{array}\right]=\left[\begin{array}{ll}
f_{11} & f_{12} \\
f_{21} & f_{22}
\end{array}\right]\left[\begin{array}{l}
Q_{\ell} \\
Q_{r}
\end{array}\right]
$$

where subscripts $\ell$ and $r$ refer to left and right cover plate surfaces, respectively, and $Q_{\ell}$ and $Q_{r}$ are the per-unit fin heat dissipations. The impedance coefficients $f_{i j}$ obtained from this analysis appear in Table 1 where the significant undefined parameters are

$$
\begin{aligned}
\Lambda=e^{m b} \quad m_{\mathrm{s}} & =\sqrt{h /\left(\delta_{\mathrm{s}} k\right)} \quad Y_{0, \mathrm{~s}}=k A m_{\mathrm{s}} \\
\gamma & =2 Z_{0} Y_{0, \mathrm{~s}} \tanh \left(m_{\mathrm{s}} b\right)
\end{aligned}
$$

and where the subscript $s$ refers to the splitter plate. Critical dimensions for both connecting fins and splitter plate are shown in the inset of Fig. $2 . S$ is the surface area of the exterior surface within the repeating cell minus the footprint created by the connecting cold plate fin defined in Table 2 as illustrated in Fig. 2. Heat loss through this left/right surface to the environment is equal to $h S \theta$. Therefore, it is correct to interpret $h S$ as the per-unit-cell cover-plate surface admittance.

Table 2: Structure Relations

\begin{tabular}{ll}
$S=(W-\delta) L$ & $b_{s}=\left[W_{T} /\left(2 N_{f}\right)-\delta\right]$ \\
$A=4\left(2 b_{s}+b\right) L$ & $V=2\left(\delta b+\delta_{s} b_{s}\right) L$ \\
\hline
\end{tabular}

In order to analyze the transient response of the structure, these results can be extended using rules that follow from a comparison of eq. (4) with eq. (17) 
expressed in terms of

$$
m^{2}=\frac{h}{\delta k} \quad \alpha=\frac{k}{c \rho} \quad \theta=T-T_{e}
$$

where the excess temperature $\theta$ is measured relative to the environment temperature. The general timeindependent solution of eq. (2) can be expressed as a linear combination of terms involving $\cosh m b$ and $\sinh m b$. Two boundary conditions will generally be required to complete a solution. For boundary conditions specified at the tip, the general solution in two-port format is:

$$
\left[\begin{array}{l}
\theta(x) \\
q(x)
\end{array}\right]=\left[\begin{array}{cc}
\cosh m x & Z_{0} \sinh m x \\
Y_{0} \sinh m x & \cosh m x
\end{array}\right]\left[\begin{array}{l}
\theta(0) \\
q(0)
\end{array}\right]
$$

where the fin's characteristic admittance $Y_{0}$ is given by:

$$
Y_{0}=Z_{0}^{-1}=k A m
$$

Appropriately, $Z_{0}$ is referred to as the fin's characteristic impedance. In order to lighten the notation subscript ' $a$ ' and ' $b$ ' will be used to specify tip and base, respectively. The conditions at the base can be obtained from eq. (3) after setting $x=b$.

$$
\left[\begin{array}{c}
\theta_{b} \\
q_{b}
\end{array}\right]=\left[\begin{array}{cc}
\cosh m b & Z_{0} \sinh m b \\
Y_{0} \sinh m b & \cosh m b
\end{array}\right]\left[\begin{array}{c}
\theta_{a} \\
q_{a}
\end{array}\right]
$$

Note that for an insulated tip, i.e., $q_{a}=0$, the input admittance is

$$
Y_{\mathrm{in}}=\frac{q_{b}}{\theta_{b}}=Y_{0} \tanh m b
$$

and this is exactly the same expression as one obtains for an electrical transmission line of length $b$ which is terminated in an open.

\section{Transient Two-Port Analysis}

For transient analysis it is assumed that at $t=0^{-}$the initial thermal conditions along the fin satisfy a static solution consistent with the boundary conditions also specified at tip and base at time $t=0^{-}$. Under these conditions a separation of the general solution $\theta(x, t)$ into a static part (or DC) and time varying time part (TV) will lead to a decoupling of the differential problem into two individually tractable parts. It follows that

$$
\begin{aligned}
& \theta(x, t)=\theta_{D C}(x)+\theta_{T V}(x, t) \\
& q(x, t)=q_{D C}(x)+q_{T V}(x, t)
\end{aligned}
$$

Based on the assumption that the system is initially in static equilibrium with boundary conditions at $t=$ $0^{-}$we have from eq. (8) and eq. (2)

$$
\frac{d^{2}}{d x^{2}} \theta_{D C}(x)-m^{2} \theta_{D C}(x)=0
$$

and

$$
\frac{d^{2}}{d x^{2}} \theta_{T V}(x, t)-m^{2} \theta_{T V}(x, t)-\frac{1}{\alpha} \frac{\partial}{\partial t} \theta_{T V}(x, t)=0
$$

Consistent with eq. (3)

$$
\theta_{D C}(x)=T_{i}(x)-T_{e}
$$

where $T_{i}(x)$ is the initial equilibrium solution immediately predictable using Section 2 methods for static analysis. In order to satisfy eq. (8a)

$$
\theta_{T V}(x, t) \equiv T(x, t)-T_{i}(x)
$$

which guarantees that $\theta_{T V}(x, 0)=0$, a condition needed to apply the single-sided Laplace transform methods. After application of the single-sided Laplace transform

$$
\mathcal{L}[f(t)]=F(s)=\int_{0^{+}}^{\infty} e^{-s t} f(t) d t
$$

to both sides of eq. (10), where $f(t) \longrightarrow \theta(x, t)$, it follows that $[1,2]$

$$
\frac{d^{2}}{d x^{2}} \hat{\theta}_{T V}(x, s)-\hat{m}^{2} \hat{\theta}_{T V}(x, s)=0
$$

where

$$
\hat{m}^{2}=\left(m^{2}+\frac{s}{\alpha}\right)
$$

The solution to (13) can be expressed in a linear combination of $\cosh \hat{m} b$ and $\sinh \hat{m} b$. After applying the following rules for calculating heat flows

$$
\begin{gathered}
q_{D C}(x)=k A \frac{d \theta_{D C}}{d x}(x) \\
\hat{q}_{T V}(x, s)=k A \frac{d \hat{\theta}_{T V}}{d x}(x, s)
\end{gathered}
$$

it is again possible to represent the solution in twoport format assuming known conditions at the tip. In particular, we find

$$
\left[\begin{array}{l}
\hat{\theta}(x, s) \\
\hat{q}(x, s)
\end{array}\right]=\left[\begin{array}{cc}
\cosh \hat{m} x & \hat{Z}_{0} \sinh \hat{m} x \\
\hat{Y}_{0} \sinh \hat{m} x & \cosh \hat{m} x
\end{array}\right]\left[\begin{array}{l}
\hat{\theta}_{a}(s) \\
\hat{q}_{a}(s)
\end{array}\right]
$$

where $s$-dependent admittances and impedances satisfy

$$
\hat{Y}_{0}=k A \hat{m}=\left(\hat{Z}_{0}\right)^{-1}
$$

Equation (17) agrees with eq. (6) for the DC case obtained by setting $s=0$. 
and eq. (5) with eq. (18). In particular, it is sufficient to let

$$
m_{s} \longrightarrow \hat{m}_{s}=m_{s} \sqrt{\left(1+\frac{s}{\alpha}\right)}
$$

and

$$
m \longrightarrow \hat{m}=m \sqrt{\left(1+\frac{s}{\alpha}\right)}
$$

which will generally affect both the admittance and impedance. The extended Laplace transform solution of eq. (19) needed for transient analysis can be represented

$$
\begin{aligned}
& \hat{\theta}_{b \ell}(s)=\hat{f}_{11}(s) \hat{Q}_{\ell}(s)+\hat{f}_{12}(s) \hat{Q}_{r}(s) \\
& \hat{\theta}_{b r}(s)=\hat{f}_{21}(s) \hat{Q}_{\ell}(s)+\hat{f}_{22}(s) \hat{Q}_{r}(s)
\end{aligned}
$$

where the form for the per-unit-fin $s$-dependent power dissipation $\hat{Q}_{\ell}(s)$ and $\hat{Q}_{r}(s)$ are determined by the driving-point time variation in the power dissipation requirements, e.g., step, ramp, sinusoid, etc. Laplace inversion will lead to the transient response associated with the coverplate excess temperatures. In principle this can be done exactly using either tables or residue theory. However, for fin arrays such as in cold plates, the expressions are too complicated for these methods. Fortunately, a numerical method for Laplace inversion known as the Fourier Series Technique has been reported [4] This method provides an accurate solution for any value of $t$ up to $2 T^{*}$. To implement the method the following calculation can be made.

$$
f(t)=\frac{e^{p t}}{T^{*}} R E\left\{\frac{F(p)}{2}+\sum_{i=1}^{N} F(p+j \omega) e^{j \omega t}\right\}
$$

where $\omega=\pi i / T^{*}$ and where $p$ is a positive real constant which meets the condition that the $p T^{*}(0.3 \times$ 15 )product is a constant roughly within the range of values between four and five [4]. $N$ can be increased to improve the accuracy. For the application under consideration $\theta_{b \ell}(t)$ and $\theta_{b r}(t)$ are obtained, respectively, using $\hat{\theta}_{b l}(s)$ and $\hat{\theta}_{b r}(s)$ for $F(s)$ in eq. (23).

The total heat dissipations, $Q_{T L}$ and $Q_{T R}$, can be calculated from the IC manufacturers specifications of the electronic components and are typically known in the design. The per unit cell power dissipations are calculated from the scaling rules

$$
\left[\begin{array}{c}
Q_{L} \\
Q_{R} \\
W
\end{array}\right] \simeq\left[\begin{array}{c}
Q_{T L} \\
Q_{T R} \\
W_{T}
\end{array}\right] / N_{f}
$$

where, as represented on Fig. 2, $N_{f}$ is the number of fins connected to either left or right exterior cover plates and $W_{T}$ is the total vertical length of the exterior surface. The fin height for the splitter plate $b_{s}$ can be calculated using the rule given in Table 2 .

\section{Example - Step Response}

In this example the double stack cold plate is assumed to be built using 11.1 plain plate aluminum. Material constants and critical dimensions are specified in column 1 of Table 3 . In column 2 of Table 3 the essential design parameters, which are derived from column 1 specifications, are cited. As specified in Table 2, the total heat loading on the left side is fixed at $150 \mathrm{~W}$ and the total heat loading on the right is assumed to vary between 1 and $250 \mathrm{~W}$. Assume that the designer has a priori established that the critical temperature limit based on electronic data sheet components is $120^{\circ} \mathrm{C}$. However, for conservative design purposes it is established that a $10^{\circ}$ safety margin be taken. The

\begin{tabular}{|c|c|c|c|}
\hline \multicolumn{2}{|c|}{$\begin{array}{c}\text { Design } \\
\text { Specifications }\end{array}$} & \multicolumn{2}{|c|}{$\begin{array}{c}\text { Design Parameters } \\
\text { (all MKS Units) }\end{array}$} \\
\hline $\begin{array}{l}T_{c} \\
T M A X \\
W_{T}=L \\
k \\
h \\
\# \text { fins/inch } \\
\delta \\
\delta_{s} \\
b \\
Q_{T L} \\
Q_{T R} \\
\rho \\
c\end{array}$ & $\begin{array}{l}=75^{\circ} \\
=110^{\circ} \\
=4.0 \text { inches } \\
=180 \mathrm{~W} / \mathrm{m}^{\circ}{ }^{\circ} \mathrm{K} \\
=72 \mathrm{~W} / \mathrm{m}^{2}-{ }^{\circ} \mathrm{K} \\
=11.1 \\
=1 / 100 \mathrm{inch} \\
=2 / 100 \mathrm{inch} \\
=1 / 4 \mathrm{inch} \\
=150 \mathrm{~W} \\
=[1,250] \mathrm{W} \\
2702 \mathrm{~kg} / \mathrm{m}^{3} \\
=903 \mathrm{~J} / \mathrm{kg}^{\circ} \mathrm{K}\end{array}$ & $\begin{array}{l}\theta_{\max } \\
N_{f} \\
W \\
m \\
m_{\theta} \\
\Lambda \\
b_{s} \\
Y_{0} \\
Y_{0 s} \\
Z_{0} \\
S \\
Y_{\text {in,s }} \\
\gamma \\
\alpha\end{array}$ & $\begin{array}{l}=35^{\circ} \\
=44.4 \\
=0.0023 \\
=56.1214 \\
=39.6838 \\
=1.4281 \\
=0.001 \\
=0.20607 \\
=0.3687 \\
=3.859 \\
=2.0668 * 10^{-4} \\
=0.0291 \\
=0.1141 \\
=73 \times 10^{-6}\end{array}$ \\
\hline
\end{tabular}
inlet temperature of the forced air flow is at $75^{\circ}$ and therefore the maximum peak excess temperature is $35^{\circ}$.

Table 3: Specifications and Parameters Used in Cold Plate Example

Consistent with the procedure for analysis the problem is split into DC and time varying points. As previously explained the $\mathrm{TV}$ responses are references to coordinations at $t=0^{-}$. For this problem we assume that the Laplace transformed RHS power dissipation associated with time varying component can be represented as

$$
\left(\hat{Q}_{r}\right)_{T V}=\frac{249}{N_{f} s}
$$

where $\mathcal{L}[U(t)]=1 / s$. It also follows that because the LHS power dissipation is fixed $\left(\hat{Q}_{\ell}\right)_{T V}=0$. The general solution eq. (25) reduces to:

$$
\begin{aligned}
& \left(\hat{\theta}_{b \ell}\right)_{T V}=\hat{f}_{12}(s)\left(\hat{Q}_{r}\right)_{T V} \\
& \left(\hat{\theta}_{b r}\right)_{T V}=\hat{f}_{22}(s)\left(\hat{Q}_{r}\right)_{T V}
\end{aligned}
$$


Substitution of eq. (25) into eq. (26), and followed by a numerical inversion of eq. (23), leads to the results shown on Fig. 3. As expected the time varying components of the solution starts at zero and (for the step input) approach asymptotic values. The horizontal lines, which apparently agree with these asymptotic values, were calculated using the difference of the static solutions corresponding to initial boundary conditions $\left(t=0^{-}\right)$and final boundary conditions (see Table 4). From Table 4 it is evident that both right and left sides will eventually exceed the $35^{\circ}$ excess temperature limit (see Table 2). From Fig. 3 it is possible to predict at what instant the temperature limits are crossed. For example, for the RHS surface the initial temperature is $14.2^{\circ}$. Therefore, at $\left(\theta_{b r}\right)_{T V}-\left(\theta_{b r}\right)_{T V}=35^{\circ}-14.2^{\circ}=20.8^{\circ}$ the RHS limit is reached which occurs after about approximately four seconds.

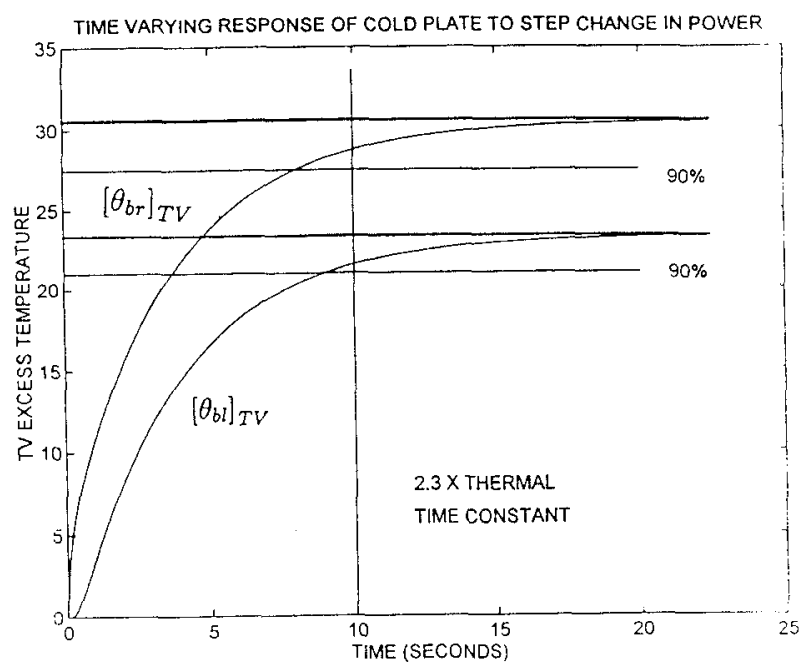

Figure 3: Time varying response of cold plate to step change in power.

Table 4: Static Analysis Predictions

\begin{tabular}{cccc}
\hline$Q_{T L}$ & $Q_{T R}$ & $\left(\theta_{b \ell}\right)_{D C}$ & $\left(\theta_{b r}\right)_{D C}$ \\
\hline & & & \\
$150 \mathrm{~W}$ & $1 \mathrm{~W}$ & $18.5^{\circ}$ & 14.2 \\
$150 \mathrm{~W}$ & $250 \mathrm{~W}$ & $41.8^{\circ}$ & 44.7 \\
\hline
\end{tabular}

An order of magnitude check on the step response shown is provided by using the thermal time constant [5] for a solid having a convective dissipating surface area, $A_{s}$, and a volume, $V$,

$$
\tau=\frac{h A_{s}}{\rho V c} \quad\left(\text { or } \frac{h A \alpha}{k V}\right)
$$

which is expressed in terms of a surface admittance $\left(h A_{s}\right)$ and a thermal capacitance $(\rho V c)$. For electrical engineers the analogy to the RC time constant should be evident and substitution demonstrates that $\tau=4.34$ sec. A first order step response will reach $90 \%$ of the peak in 2.3 time constants and for reference this value is indicated on Fig. 3 by a vertical line. Horizontal lines corresponding to $90 \%$ of limiting $\left(\theta_{b r}\right)_{T V}$ and $\left(\theta_{b \ell}\right)_{T V}$ are also shown for reference. The first order estimate is a useful approximate gauge for the rise time response of the more complicated system, providing the desired order of magnitude check.

\section{Conclusion}

A model for predicting the transient response for complicated fin structures has been presented. Cold plate structures often used to keep surface electronic components with the manufacturers temperature specifications serves as a practical example. It is not difficult to imagine advanced highly efficient automatic control cooling systems which automatically adjust via feedback (e.g., using a thermocouple), to the heat loads. In order to predict the temperature dynamics during heat load transitions, a model such as described here, would be essential.

\section{References}

[1] A. D. Kraus, "Analysis of Extended Surfaces," J. Heat Transfer, Vol. 110, pp. 1071-1081, Nov. 1988.

[2] N. V. Suryanarayana, "Transient Response of Straight Fins, Part II," Trans. ASME, PP. 324326, May 1976.

[3] R. J. Pieper and A. D. Kraus, "Performance Analysis of Double Stack Cold Plates Covering All Conditions of Asymmetric Heat Loading," $J$. Electronic Packaging, in review.

[4] H-S. Chu, C-K. Chen, C-I. Weng, "Applications of Fourier Series Technique to Transient Heat Transfer Problem," Chem. Eng. Commun., Vol. 16, pp. 215-225, 1982.

[5] F. Kreith and W. Z. Black, Basic Heat Transfer, Harper \& Row Publishers, New York, 1980. 